\title{
Co-design of dynamic allocation functions and anti-windup*
}

\author{
Thiago A. Lima ${ }^{\dagger 1,2}$, Sophie Tarbouriech ${ }^{\ddagger 1}$, Fabrício G. Nogueira ${ }^{\S 1}$ and Bismark C. Torrico ${ }^{\mathbb{2} 2}$ \\ ${ }^{1}$ LAAS-CNRS, Université de Toulouse, CNRS, Toulouse, France \\ ${ }^{2}$ Department of Electrical Engineering, Federal University of Ceará, Fortaleza, Brazil
}

October 14, 2020

\begin{abstract}
This paper addresses the design of dynamic allocation functions for systems with saturating actuators. The allocator can redistribute the desired control effort within the multiple actuators by penalizing each actuator to be more or less used, while also taking into account a criterium for minimization of their total energy consumption over time. Anti-windup gains are added to both the controller and the dynamic allocator state to deal with the saturation condition. Convex conditions for the co-design of both the dynamic allocator and anti-windup gains are expressed in the form of linear matrix inequalities (LMIs). Such conditions allow to deal with the multiple objective problems of enlarging the estimates of the basin of attraction and minimizing the total energy consumption of the actuators. Two examples borrowed from the literature illustrate the proposed technique and show its effectiveness.
\end{abstract}

Index Terms - Dynamic Allocation, Saturating Actuators, Anti-windup, LMIs.

\section{Introduction}

Control allocation is widely used in over-actuated systems and consists of applying some algorithm to distribute the computed control effort throughout the multiple actuators that together drive the plant states and/or outputs. The control allocation problem is found in numerous applications and occurs, in general, when the regulated plant contains torques and/or forces as inputs, which in turn are generated by a set of multiple actuators (for example microthrusters in space applications) that jointly produce the desired control effort. As pointed out in [1,2], the advantages with the use of the control allocation approach are, in general, modularity and ability to handle constraints as the redundancy of actuators allows multiple optimal criteria to be taken into account.

The control allocation problem is treated in several papers dealing with specific applications, in particular in the aeronautical or spatial contexts: see, for example, the works of [3], [4], [5], [6]. Technical solutions are also proposed in the literature from a theoretical point of view. Both the works in [7] and [8] consider the output regulation problem of over-actuated systems in the presence of full information regarding the system states and exogenous inputs. More specifically, [7] proposes an allocation mechanism that takes the form of a hybrid system and accounts for input constraints. In [9], optimization-based algorithms, as interior point method, are detailed to compute optimal allocation given the actuators constraints. The online implementation of this kind of technique can be, however, computationally expensive, while stability

\footnotetext{
*This study was financed in part by the Coordenação de Aperfeiçoamento de Pessoal de Nível Superior - Brasil (Capes) Finance Code 001, and by ANR project HANDY 18-CE40-0010.

†talveslima@laas.fr,thiago.lima@alu.ufc.br

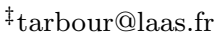

§fnogueira@dee.ufc.br

I bismark@dee.ufc.br
} 
analysis of the closed loop is not straightforward. The presence of constraints inherently causes errors between the desired control effort and the actual plant input, which can lead to poor response and even instability. In this context, Lyapunov-based approaches with guarantees of stability for the constrained closed-loop system have also been searched, for example in [10-13]. In this vein, the work in [14] proposed the use of an allocation function and anti-windup designed with an optimization procedure that envisaged the minimization of this error while guaranteeing closed-loop stability. Nonetheless, the proposed allocation format and design procedure did not take into account other important issues as the ability to penalize the use of the different actuators and energy consumption minimization.

The work in [15] formally defines the concepts of weak and strong redundancy, in which the former implies that multiple actuators can induce the same steady-state value for the plant output while the latter implies that they can also impose equal trajectories. The use of a dynamic allocator system between the controller and the plant is then proposed with the goal to distribute the control effort by penalizing the use of the multiple actuators. The paper also deals with the cases of input and rate saturation of the actuators. The use of dynamic allocation functions was shown to be a good alternative in terms of both computational effort and robustness. Nonetheless, some important issues regarding the strategy can be shortlisted: i) [15] does not consider the co-design problem of the anti-windup loop and allocation. ii) The parameters of the allocator in [15] are manually selected. iii) The formulation in [15] is focused in the specific case where the size of the plant input (noted $m_{c}$ in the paper) is equal to the size of allocator output (noted $m_{a}$ in the paper) and the influence matrix (noted $\mathrm{M}$ in the paper) is the identity matrix (i.e., $\mathrm{M}=\mathrm{I}$ ). In the current work, we can deal with a broader range of systems since we consider the case of $m_{a} \geq m_{c}$. iv) In the regulatory case (null external references) with actuator saturation, the states of the allocator do not asymptotically converge to the origin, which causes waste of energy in the actuators.

Indeed, this paper aims at proposing a more general allocator function format and design method that solve the above-described problems. By considering a Lyapunov-based approach, theoretical conditions are derived in terms of linear matrix inequalities (LMIs) in order to solve the co-design of the allocator and anti-windup loop. Furthermore, an optimization scheme consisting of the minimization of the energy consumption in the actuators and maximization of the closed-loop region of attraction is proposed. As in [15], the use of the actuators can also be penalized according to the user desire. Some ideas from [14] are also recovered to show that the allocator is somehow effective concerning the allocation error between the computed control effort and the actual plant input. The use in two examples borrowed from the literature shows the effectiveness of the developed strategy.

Notation. For a matrix $\mathrm{Y}$ in $\mathbb{R}^{n \times m}, \mathrm{Y}^{\top}$ in $\mathbb{R}^{m \times n}$ means its transpose, $\mathrm{Y}_{(i)}$ denotes its $i$ th row, while for $v$ in $\mathbb{R}^{m}, v_{(i)}$ denotes its $i$ th component. For matrices $\mathrm{W}=\mathrm{W}^{\top}$ and $\mathrm{Z}=\mathrm{Z}^{\top}$ in $\mathbb{R}^{n \times n}, \mathrm{~W} \succ \mathrm{Z}$ means that $\mathrm{W}-\mathrm{Z}$ is positive definite. Likewise, $\mathrm{W} \succeq \mathrm{Z}$ means that $\mathrm{W}-\mathrm{Z}$ is positive semi-definite. $\mathbb{S}_{n}^{+}$stands for the set of symmetric positive definite matrices in $\mathbb{R}^{n \times n}$. I and 0 denote identity and null matrices of appropriate dimensions, although their dimensions can be explicitly presented whenever relevant. The $\star$ in $\left[\begin{array}{cc}\mathrm{A} & \mathrm{B} \\ \star & \mathrm{C}\end{array}\right]$ denotes symmetric blocks, that is $\star=\mathrm{B}^{\top}$. Finally, for matrices $\mathrm{W}$ and $\mathrm{Z}, \operatorname{diag}(\mathrm{W}, \mathrm{Z})$ corresponds to the block-diagonal matrix.

\section{Problem formulation}

\subsection{General view}

Consider the general view of the control allocation problem shown in Fig. 1.

Subsystems $\mathcal{C}, \mathcal{F}$, and $\mathcal{P}$ are the controller, the control allocator, and the plant, respectively, while $\mathrm{M}$ is the influence matrix. The plant is driven by $u_{p}$ in $\mathbb{R}^{m_{c}}$ inputs. The controller computes a set of desired $y_{c}$ in $\mathbb{R}^{m_{c}}$ efforts that must be injected in the plant in ideal conditions. The plant input is generated by a set of $m_{a} \geq m_{c}$ actuators, represented by the signal $y_{f}$ in $\mathbb{R}^{m_{a}}$. The plant input is given by $u_{p}=\operatorname{Msat}\left(y_{f}\right)$ with the decentralized saturation function being defined as

$$
\operatorname{sat}\left(y_{f(i)}\right)=\operatorname{sign}\left(y_{f(i)}\right) \min \left\{\left|y_{f(i)}\right|, \bar{u}_{(i)}\right\}, \bar{u}_{(i)}>0,
$$




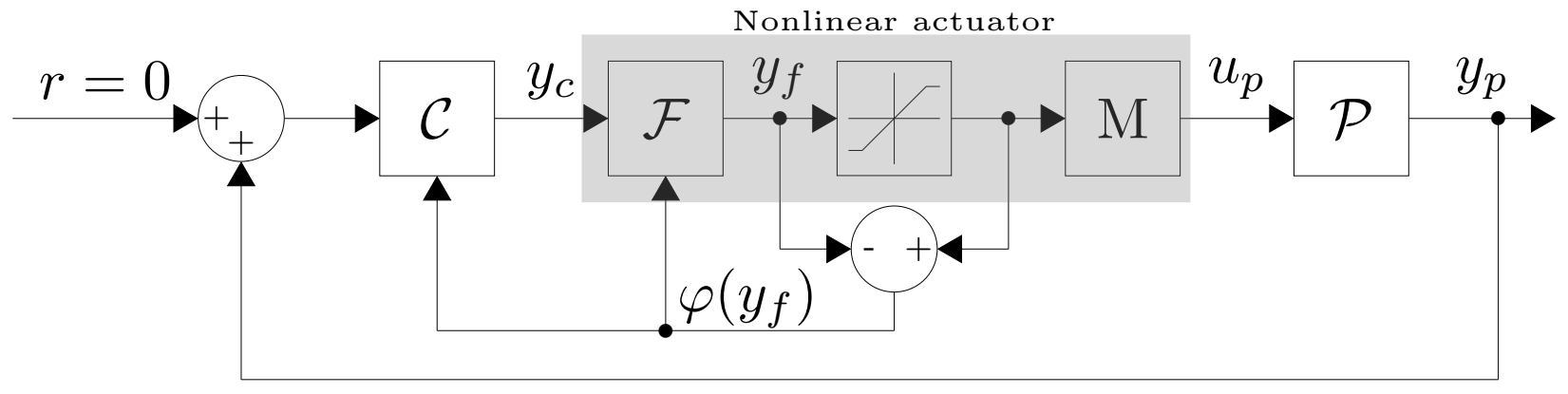

Figure 1: General view of control allocation problem.

for $i=1, \ldots, m_{a}$, where $\bar{u}_{(i)}$ denotes the amplitude bound in each actuator, and the so-called influence matrix $\mathrm{M}$ in $\mathbb{R}^{m_{c} \times m_{a}}$ maps how each individual effort of the $m_{a}$ actuators combines to generate the inputs acting on the plant. Furthermore, in this paper we consider the regulatory case, i.e. $r=0$.

The simplest allocation function often considered in the literature is given by the right pseudo-inverse of $\mathrm{M}$, that is, $\mathcal{F}=\mathrm{M}^{\dagger}$, with $\mathrm{MM}^{\dagger}=\mathrm{I}$. In the case the actuator is not subject to saturation or other nonlinearities, this allocator is able to guarantee stability of the closed loop since the interconnection is given by $u_{p}=\mathrm{MM}^{\dagger} y_{c}=y_{c}$, and therefore no error between $u_{p}$ and $y_{c}$ is produced. However:

i. This popular static allocator is not able to take advantage of the multi-actuated nature of the system to redistribute the effort among desired actuators. This is important, for example, in case of actuator failures and/or operational constraints that demand certain actuators to be more or less used than others to produce the desired control effort.

ii. In the presence of nonlinearities as the actuator saturation, guarantees of stability of the closed loop, as well as estimation of regions of safe operation, need to be assured. In this context, more complex allocation functions with the ability to handle redundancy and constraints should be applied.

\subsection{Plant and controller description}

Consider the plant $\mathcal{P}$ described by the following equations

$$
\mathcal{P} \sim\left\{\begin{array}{l}
\dot{x}_{p}=\mathrm{A}_{p} x_{p}+\mathrm{B}_{p} u_{p}, \\
y_{p}=\mathrm{C}_{p} x_{p},
\end{array}\right.
$$

where $x_{p}$ in $\mathbb{R}^{n_{p}}$ is the plant state vector, $u_{p}$ in $\mathbb{R}^{m_{c}}$ is the plant input, $y_{p}$ in $\mathbb{R}^{q}$ is the measured output. $\mathrm{A}_{p}$, $\mathrm{B}_{p}$, and $\mathrm{C}_{p}$ are all constant and known matrices of appropriate dimensions. Furthermore, the pairs $\left(\mathrm{A}_{p}, \mathrm{~B}_{p}\right)$ and $\left(\mathrm{C}_{p}, \mathrm{~A}_{p}\right)$ are supposed to be controllable and observable.

Let us assume that the plant (2) is stabilized by a dynamic output controller $\mathcal{C}$ linearly designed via the connection $u_{p}=y_{c}$, that is without taking into account the saturation and with $\mathcal{F}=\mathrm{M}^{\dagger}$. The controller $\mathcal{C}$ is defined by the following equations

$$
\mathcal{C} \sim\left\{\begin{array}{l}
\dot{x}_{c}=\mathrm{A}_{c} x_{c}+\mathrm{B}_{c} y_{p}+v_{a w}, \\
y_{c}=\mathrm{C}_{c} x_{c}+\mathrm{D}_{c} y_{p},
\end{array}\right.
$$

where $x_{c}$ in $\mathbb{R}^{n_{c}}$ is the controller state vector and $y_{c}$ in $\mathbb{R}^{m_{c}}$ is the controller output. $\mathrm{A}_{c}, \mathrm{~B}_{c}, \mathrm{C}_{c}$ and $\mathrm{D}_{c}$ are supposed known. The anti-windup compensation signal $v_{a w}$ is added in order to mitigate the undesired effects of saturation (see, for example, [16], [17]). In this paper, we consider the anti-windup signal $v_{a w}=\mathrm{E}_{c} \varphi\left(y_{f}\right)$, $\mathrm{E}_{c}$ in $\mathbb{R}^{n_{c} \times m_{a}}$, with the deadzone $\varphi\left(y_{f}\right)$ defined as

$$
\varphi\left(y_{f}\right)=\operatorname{sat}\left(y_{f}\right)-y_{f}
$$


where the saturation map is defined from (1) and $y_{f}$ is the output of the allocation function.

Remark 1. By construction, the linear connection plant-controller is supposed to be stable. In other words, the controller (3) (with $v_{a w}=0$ ) stabilizes the plant (2) through the linear interconnection $u_{p}=y_{c}$ and therefore the matrix

$$
\mathrm{A}_{0}=\left[\begin{array}{cc}
\mathrm{A}_{p}+\mathrm{B}_{p} \mathrm{D}_{c} \mathrm{C}_{p} & \mathrm{~B}_{p} \mathrm{C}_{c} \\
\mathrm{~B}_{c} \mathrm{C}_{p} & \mathrm{~A}_{c}
\end{array}\right] \text { in } \mathbb{R}^{\left(n_{p}+n_{c}\right) \times\left(n_{p}+n_{c}\right)}
$$

is Hurwitz.

\subsection{Dynamic allocation function description}

Consider the influence matrix $\mathrm{M}$ in $\mathbb{R}^{m_{c} \times m_{a}}$ in the case $m_{a}>m_{c}$, supposedly full row rank. Let $\mathrm{N}$ in $\mathbb{R}^{m_{a} \times n_{f}}, n_{f}=m_{a}-m_{c}$, be a basis for the Kernel of $\mathrm{M}$, i.e. $\mathrm{MN}=0$. We then propose the following dynamic allocation function

$$
\mathcal{F} \sim\left\{\begin{array}{l}
\dot{x}_{f}=\mathrm{K}_{f} \mathrm{~N}^{\top} \mathrm{WN} x_{f}+\mathrm{K}_{f} \mathrm{~N}^{\top} \mathrm{WM}^{\dagger} y_{c}+\mathrm{E}_{f} \varphi\left(y_{f}\right), \\
y_{f}=\mathrm{N} x_{f}+\mathrm{M}^{\dagger} y_{c}
\end{array}\right.
$$

where $x_{f}$ in $\mathbb{R}^{n_{f}}$ is the allocator state vector, and $y_{f}$ in $\mathbb{R}^{m_{a}}$ is the allocator output. $\mathrm{W}=\operatorname{diag}\left(w_{1}, w_{2}, \ldots, w_{m_{a}}\right)$ in $\mathbb{S}_{m_{a}}^{+}$is a matrix which receives the weightings that penalizes the use of each actuator. Matrices $\mathrm{K}_{f}$ in $\mathbb{R}^{n_{f} \times n_{f}}$ and $\mathrm{E}_{f}$ in $\mathbb{R}^{n_{f} \times m_{a}}$ must be designed to achieve desired behavior of the allocator by taking into account the presence of saturation. This allocation format is particularly interesting since it is in some sense optimal in terms of both the allocation error and actuators usage, as explained in the next two remarks.

Remark 2. Consider the general expression $y_{f}=\mathrm{C}_{f} x_{f}+\mathrm{D}_{f} y_{c}$, and let us define the allocator error as $e=u_{p}-y_{c}$. Then using the definition of $\varphi\left(y_{f}\right)$ in (4), the expression $e=\left(\mathrm{MD}_{f}-\mathrm{I}\right) y_{c}+\mathrm{MC}_{f} x_{f}+\mathrm{M} \varphi\left(y_{f}\right)$ is easily obtained. It is straightforward to see that the choice $\mathrm{D}_{f}=\mathrm{M}^{\dagger}, \mathrm{C}_{f}=\mathrm{N}$ leads to $e=\mathrm{M} \varphi\left(y_{f}\right)$, therefore the error is null in absence of saturation. Furthermore, by guaranteeing convergence of the extended vector $x=\left[\begin{array}{lll}x_{p}^{\top} & x_{c}^{\top} & x_{f}^{\top}\end{array}\right]^{\top}$ to the origin, we always obtain $e^{*}=0$, where $e^{*}$ is the steady-state value of $e$.

Remark 3. Consider the cost function

$$
\min _{x_{f}} \mathrm{~T}\left(y_{f}\right)=y_{f}^{\top} \mathrm{W} y_{f} \text { subject to } y_{f}=\mathrm{N} x_{f}+\mathrm{M}^{\dagger} y_{c}^{*} \text {, }
$$

where $y_{c}^{*}$ is any controller output. The optimal solution to (7) is given by $x_{f}=-\left(\mathrm{N}^{\top} \mathrm{W}^{\top} \mathrm{N}\right)^{-1} \mathrm{~N}^{\top} \mathrm{WM}^{\dagger} y_{c}^{*}$, which corresponds to the steady-state value of $x_{f}$ in (6).

Remark 4 (Case when $m_{a}=m_{c}$ and $\mathrm{M}=\mathrm{I}$ ). In some papers, as the one in [15], the influence matrix $\mathrm{M}$ enters the plant model. In this case, $m_{a}=m_{c}$, the system has more inputs than states $\left(m_{c}>n_{p}\right)$ and the input-redundancy nature of the plant is explicit. All the results in this paper can straightforwardly be applied in this case by making $\mathrm{M}=\mathrm{I}$ and choosing $\mathrm{N}$ as a base for the null space of $\mathrm{B}_{p}$, that is, $\mathrm{B}_{p} \mathrm{~N}=0$. Although in this case $\mathrm{MN} \neq 0$, convergence of the allocator error to zero takes place due to the fact that the allocator states $x_{f}$ converges to zero at steady-state, thus the allocator recovers the property $u_{p}=y_{c}$ after some time.

\subsection{Closed-loop system and problem formulation}

By taking into account the definitions of $\mathcal{P}, \mathcal{C}, \mathcal{F}$, the definition of $\varphi\left(y_{f}\right)$ in $(4)$ and the connection

$u_{p}=\operatorname{Msat}\left(y_{f}\right)$, the complete closed-loop system with $x=\left[\begin{array}{lll}x_{p}^{\top} & x_{c}^{\top} & x_{f}^{\top}\end{array}\right]^{\top}$ in $\mathbb{R}^{n}, n=n_{p}+n_{c}+n_{f}$, can be written as

$$
\left\{\begin{array}{l}
\dot{x}=\left(\mathrm{A}+\mathrm{L}_{f} \mathrm{~K}_{f} \overline{\mathrm{C}}\right) x+(\mathrm{B}+\mathrm{LE}) \varphi\left(y_{f}\right) \\
y_{f}=\mathrm{C} x
\end{array}\right.
$$


where

$$
\mathrm{A}=\left[\begin{array}{cc}
\mathrm{A}_{0} & 0 \\
0 & 0
\end{array}\right], \mathrm{B}=\left[\begin{array}{c}
\mathrm{B}_{p} \mathrm{M} \\
0
\end{array}\right], \mathrm{E}=\left[\begin{array}{c}
\mathrm{E}_{c} \\
\mathrm{E}_{f}
\end{array}\right], \mathrm{L}=\left[\begin{array}{cc}
0_{n_{p} \times n_{c}} & \overbrace{0_{n_{p} \times n_{f}}}^{\mathrm{L}_{f}=} \\
\mathrm{I}_{n_{c}} & 0_{n_{c} \times n_{f}} \\
0_{n_{f} \times n_{c}} & \mathrm{I}_{n_{f}}
\end{array}\right], \mathrm{C}=\left[\begin{array}{lll}
\mathrm{M}^{\dagger} \mathrm{D}_{c} \mathrm{C}_{p} & \mathrm{M}^{\dagger} \mathrm{C}_{c} & \mathrm{~N}
\end{array}\right], \overline{\mathrm{C}}=\mathrm{N}^{\top} \mathrm{WC},
$$

with $\mathrm{A}_{0}$ defined in (5). The presence of the deadzone in the closed-loop dynamics (8) implies to characterize a suitable region of the state space in which the stability is ensured (see, for example, [16], [17]). In general the global asymptotic stability of the origin (that is for any initial condition $x(0)$ in $\mathbb{R}^{n}$ ) does not hold except if the open loop has suitable properties of stability [18]. Hence, the regional stability (that is, only for initial conditions in a neighborhood of the origin) has to be studied. Since exact characterization of the basin of attraction of the origin remains an open problem, a challenging problem consists in providing an estimate of the basin of attraction as accurate as possible.

Furthermore, we want to ensure some level of performance to the allocator in terms of the total energy consumption of the actuators, which can be done by imposing conditions that limit the energy of the signal $s a t\left(y_{f}\right)$. With respect to (3) and (6), the main objective of this paper is to propose the co-design of the dynamic allocation function, that is $\mathrm{K}_{f}$, and $\mathrm{E}_{f}$, along with the controller anti-windup gain $\mathrm{E}_{c}$. Then the problem we intend to solve can be summarized as follows.

Problem 1. Given the controller matrices $\mathrm{A}_{c}, \mathrm{~B}_{c}, \mathrm{C}_{c}, \mathrm{D}_{c}$, and the weighting matrix $\mathrm{W}$, design matrices $\mathrm{K}_{f}$, $\mathrm{E}_{f}$ and $\mathrm{E}_{c}$, such that

$i$. the regional asymptotic stability of the closed-loop system (8) is ensured and the estimate of the region of attraction is maximized.

ii. the total energy consumption of the actuators over time is minimized.

\section{$3 \quad$ Main results}

In this section, we present convex conditions for the co-design of the dynamic allocator function and the anti-windup gains. Theoretical preliminaries are initially reviewed, where we recall two important Lemmas from the literature.

\subsection{Theoretical preliminaries}

Consider an auxiliary vector $\theta$ in $\mathbb{R}^{m_{a}}$ and the set

$$
\mathcal{L}=\left\{y_{f}, \theta ;\left|y_{f(i)}-\theta_{(i)}\right| \leq \bar{u}_{(i)}, i=1, \ldots, m_{a}\right\},
$$

and recall the following Lemma from [16].

Lemma 1. [Generalized sector condition] If $y_{f}$ and $\theta$ belong to set $\mathcal{L}$, then the deadzone nonlinearity $\varphi\left(y_{f}\right)$ satisfies the following inequality, which is true for any diagonal matrix $\mathrm{S}$ in $\mathbb{S}_{m_{a}}^{+}$

$$
\varphi^{\top}\left(y_{f}\right) \mathrm{S}^{-1}\left[\varphi\left(y_{f}\right)+\theta\right] \leq 0 .
$$

Another important result widely known in the literature is re-enunciated next (see, for example, [19]).

Lemma 2. [Finsler's Lemma] Consider $\zeta$ in $\mathbb{R}^{n}, \Upsilon=\Upsilon^{\top}$ in $\mathbb{R}^{n \times n}$, and $\Gamma$ in $\mathbb{R}^{m \times n}$. The following facts are equivalent:

i. $\zeta^{\top} \Upsilon \gamma<0, \forall \zeta$ such that $\Gamma \zeta=0, \zeta \neq 0$.

ii. $\exists \mathfrak{I}$ in $\mathbb{R}^{n \times m}$ such that $\Upsilon+\mathfrak{I} \Gamma+\Gamma^{\top} \mathfrak{I}^{\top} \prec 0$. 


\subsection{Design of the allocator and anti-windup}

The following theorem provides a solution to Problem 1.

Theorem 1. Assume the existence of matrices $\overline{\mathrm{P}}$ in $\mathbb{S}_{n}^{+}, \mathrm{J}_{o}$ in $\mathbb{R}^{\left(n_{p}+n_{c}\right) \times\left(n_{p}+n_{c}\right)}, \mathrm{J}_{f}$ in $\mathbb{R}^{n_{f} \times n}, \overline{\mathrm{K}}_{f}$ in $\mathbb{R}^{n_{f} \times n_{f}}$, $\mathrm{K}_{e}$ in $\mathbb{R}^{\left(n_{c}+n_{f}\right) \times m_{a}}, \overline{\mathrm{G}}$ in $\mathbb{R}^{m_{a} \times n}$, diagonal matrix $\mathrm{S}=\mathrm{S}^{\top}$ in $\mathbb{S}_{m_{a}}^{+}$and positive scalar $\gamma$ such that

$$
\begin{aligned}
& \Psi=\left[\begin{array}{cccc}
-\overline{\mathrm{J}}-\overline{\mathrm{J}}^{\top} & \overline{\mathrm{P}}+\mathrm{A}^{\top}+\mathrm{Z}-\overline{\mathrm{J}} & \Psi_{13} & 0 \\
\star & \mathrm{A} \overline{\mathrm{J}}^{\top}+\mathrm{Z}+\overline{\mathrm{J}} \mathrm{A}^{\top}+\mathrm{Z}^{\top} & \Psi_{23} & \overline{\mathrm{J}} \mathrm{C}^{\top} \mathrm{W}^{\frac{1}{2}} \\
\star & \star & -2 \mathrm{~S} & \mathrm{SW}^{\frac{1}{2}} \\
\star & \star & \star & -\gamma \mathrm{I}
\end{array}\right] \prec 0 \\
& {\left[\begin{array}{cc}
\overline{\mathrm{P}} & \overline{\mathrm{G}}_{(i)}^{\top} \\
\star & \bar{u}_{(i)}^{2}
\end{array}\right] \succeq 0,}
\end{aligned}
$$

hold with $\Psi_{13}=\mathrm{BS}+\mathrm{LK}_{e}, \Psi_{23}=\Psi_{13}+\overline{\mathrm{G}}^{\top}-\overline{\mathrm{J}}^{\top}$ and where $\overline{\mathrm{J}}=\left[\begin{array}{ll}\overline{\mathrm{C}}^{\perp} \mathrm{J}_{o}^{\top} & \mathrm{J}_{f}^{\top}\end{array}\right]^{\top}$ in $\mathbb{R}^{n \times n}, \overline{\mathrm{C}}^{\perp}$ in $\mathbb{R}^{n \times\left(n_{p}+n_{c}\right)}$ is a matrix such that $\overline{\mathrm{C}} \overline{\mathrm{C}}^{\perp}=0$, and $\mathrm{Z}=\operatorname{diag}\left(0_{n_{p}+n_{c}}, \overline{\mathrm{K}}_{f}\right)$. Then, matrices $\mathrm{E}=\left[\begin{array}{ll}\mathrm{E}_{c}^{\top} & \mathrm{E}_{f}^{\top}\end{array}\right]^{\top}=\mathrm{K}_{e} \mathrm{~S}^{-1}$, $\mathrm{K}_{f}=\overline{\mathrm{K}}_{f}\left(\overline{\mathrm{C}} \mathrm{J}_{f}^{\top}\right)^{-1}$ are solution to Problem 1. In other words:

i. the closed-loop system (8) is asymptotically stable in the ellipsoid $\varepsilon(\mathrm{P}, 1)=\left\{x\right.$ in $\left.\mathbb{R}^{n} ; x^{\top} \mathrm{P} x \leq 1\right\}$, with $\mathrm{P}=\mathrm{J} \overline{\mathrm{P}} \mathrm{J}^{\top}$ and $\mathrm{J}=\overline{\mathrm{J}}^{-1}$;

ii. the energy of the actuators usage signal is limited and given by $\int_{0}^{\infty} \operatorname{sat}\left(y_{f}(\tau)\right)^{\top} \mathrm{W} \operatorname{sat}\left(y_{f}(\tau)\right) d \tau \leq \gamma$.

Proof. Note first that the satisfaction of inequality (11) means that matrice $\overline{\mathrm{J}}$ is non-singular. Despite the structure imposed to $\overline{\mathrm{J}}$, one can prove that there always exist $\mathrm{J}_{o}$ and $\mathrm{J}_{f}$ making $\overline{\mathrm{J}}$ non singular by using the fact that $\operatorname{rank}\{\overline{\mathrm{C}}\}=n_{f}$ and $\overline{\mathrm{C}}^{\perp}$ is the orthogonal complement of $\overline{\mathrm{C}}^{\top}$.

Consider then a quadratic Lyapunov function $\mathrm{V}(x)=x^{\top} \mathrm{P} x$, with $\mathrm{P}>0 \mathrm{in} \mathbb{S}_{n}^{+}$. The satisfaction of relation (12) ensures the inclusion of the ellipsoid $\varepsilon(\mathrm{P}, 1)=\left\{x\right.$ in $\left.\mathbb{R}^{n} ; x^{\top} \mathrm{P} x \leq 1\right\}$ in the set given by $\mathcal{L}=\{x$ in $\left.\mathbb{R}^{n} ;-\bar{u}_{(i)} \leq \mathrm{G}_{(i)} x \leq \bar{u}_{(i)}\right\}$, where $\overline{\mathrm{G}}=\mathrm{G}^{\bar{J}^{\top}}, \mathrm{P}=\overline{\mathrm{JP}} \mathrm{J}^{\top}$ and $\mathrm{J}=\overline{\mathrm{J}}^{-1}$. By choosing $\theta=\mathrm{C} x-\mathrm{G} x$, Lemma 1 applies and one gets $-2 \varphi^{\top}\left(y_{f}\right) \mathrm{S}^{-1}\left[\varphi\left(y_{f}\right)+\theta\right] \geq 0$, for any $x$ in $\mathcal{L}$. Next we want to verify that $\dot{\mathrm{V}}(x)<0$. Hence, if we verify that

$$
\dot{\mathrm{V}}(x)-2 \varphi^{\top}\left(y_{f}\right) \mathrm{S}^{-1}\left[\varphi\left(y_{f}\right)+\theta\right]+\gamma^{-1} \operatorname{sat}\left(y_{f}\right)^{\top} \mathrm{W} \operatorname{sat}\left(y_{f}\right)<0,
$$

with $\mathrm{S} \succ 0$, then $\dot{\mathrm{V}}(x)<0$, and stability of (8) for any $x$ in $\varepsilon(\mathrm{P}, 1) \subseteq \mathcal{L}$ is assured. Consequently, $\dot{\mathrm{V}}(x)+\gamma^{-1} \operatorname{sat}\left(y_{f}\right)^{\top} \mathrm{W} s a t\left(y_{f}\right)<0$ is also satisfied, which can be integrated leading to

$$
\gamma^{-1} \int_{0}^{\infty} \operatorname{sat}\left(y_{f}(\tau)\right)^{\top} \mathrm{W} \operatorname{sat}\left(y_{f}(\tau)\right) d \tau<\mathrm{V}(x(0)) \leq 1,
$$

which leads to item ii) of Theorem 1.

By using (4) and defining the augmented vector $\zeta=\left[\begin{array}{lll}\dot{x}^{\top} & x^{\top} & \varphi\left(y_{f}\right)^{\top}\end{array}\right]^{\top}$, we can rewrite inequality (13) as $\zeta^{\top} \Upsilon \zeta<0$, with the matrix $\Upsilon$ given by:

$$
\left[\begin{array}{ccc}
0 & \mathrm{P} & 0 \\
\star & \mathrm{C}^{\top} \mathrm{W}^{\frac{1}{2}} \gamma^{-1} \mathrm{~W}^{\frac{1}{2}} \mathrm{C} & (\mathrm{G}-\mathrm{C})^{\top} \mathrm{S}^{-1}+\mathrm{C}^{\top} \mathrm{W}^{\frac{1}{2}} \gamma^{-1} \mathrm{~W}^{\frac{1}{2}} \\
\star & \star & \mathrm{W}^{\frac{1}{2}} \gamma^{-1} \mathrm{~W}^{\frac{1}{2}}-2 \mathrm{~S}^{-1}
\end{array}\right] .
$$

We also have that the relation $\Gamma \zeta=0$ holds for

$$
\Gamma=\left[\begin{array}{lll}
-\mathrm{I} & \mathrm{A}+\mathrm{L}_{f} \mathrm{~K}_{f} \overline{\mathrm{C}} & \mathrm{B}+\mathrm{LE}
\end{array}\right] .
$$


From Lemma 2, by considering $\mathfrak{I}=\left[\begin{array}{lll}J^{\top} & J^{\top} & 0\end{array}\right]^{\top}$, we obtain the new condition $\bar{\Psi}=\Upsilon+\mathfrak{I} \Gamma+\Gamma^{\top} \mathfrak{I}^{\top} \prec 0$. By applying a Schur complement to $\bar{\Psi}$, followed by pre- and post-multiplying by $\operatorname{diag}\left(\mathrm{J}^{-1}, \mathrm{~J}^{-1}, \mathrm{~S}, \mathrm{I}\right)$ and its transpose, respectively, and making changes of variable $\overline{\mathrm{J}}=\mathrm{J}^{-1}, \overline{\mathrm{P}}=\overline{\mathrm{JP}} \overline{\mathrm{J}}^{\top}, \overline{\mathrm{G}}=\mathrm{G} \overline{\mathrm{J}}^{\top}, \overline{\mathrm{K}}_{f}=\mathrm{K}_{f} \overline{\mathrm{C}} \mathrm{J}_{f}^{\top}, \mathrm{K}_{e}=\mathrm{ES}$ we obtain condition $\Psi<0$.

Hence, it follows that if relations (11) and (12) are satisfied then (13) is also satisfied, or equivalently $\dot{\mathrm{V}}(x)<0$, for any $x$ in $\varepsilon(\mathrm{P}, 1)$. Then the two items of Theorem 1 are proven and the proof is completed.

Similarly to the problem of SOF (static output feedback) design, the presence of the term $\overline{\mathrm{C}}$ in $\mathbb{R}^{n_{f} \times n}$ multiplying $\mathrm{K}_{f}$ could complicate the gathering of convex conditions for the computation of the unknown variables. However, the adoption of $\overline{\mathrm{J}}=\left[\begin{array}{lll}\overline{\mathrm{C}}^{\perp} \mathrm{J}_{o}^{\top} & \mathrm{J}_{f}^{\top}\end{array}\right]^{\top}$ allows to use a change of variables to solve this problem, where $\overline{\mathrm{C}}^{\perp}$ is the orthogonal complement of $\overline{\mathrm{C}}^{\top}$ and satisfies $\left[\begin{array}{ll}\overline{\mathrm{C}}^{\perp} & \overline{\mathrm{C}}^{\top}\end{array}\right]$ square and nonsigular, and $\overline{\mathrm{C}} \overline{\mathrm{C}}^{\perp}=0$. Note that satisfaction of inequality (11) implies that $\overline{\mathrm{C}} \mathrm{J}_{f}^{\top}$ is non-singular, allowing the computation of $\mathrm{K}_{f}$. This trick only works because matrix $\overline{\mathrm{C}}$ is full rank due to the term $\mathrm{N}^{\top} \mathrm{WN}$ and the closed-loop matrix A does not contain any exponentially unstable eigenvalues since the sub-matrix $\mathrm{A}_{0}$ is Hurwitz by definition (Remark 1). Therefore, it is important to freeze that this solution cannot be applied to the general case of SOF design where the plant dynamics might include unstable poles.

Remark 5 (On the choice of matrix W). It can be noted from Remark 3 and item ii) of Theorem 1 that the entries of the matrix $\mathrm{W}$ are inversely proportional to the level of usage of the actuator. Although the user can specify any desired value $w_{i}>0$, one promising choice in the case the level of saturation of the different actuators is different is to make $w_{i}=\bar{u}_{(i)}^{-1}$.

Remark 6. In case the plant state matrix $\mathrm{A}_{p}$ is Hurwitz stable, global stability of the closed loop can be achieved and the design of $\mathrm{K}_{f}, \mathrm{E}_{f}, \mathrm{E}_{c}$ can also be realized by solving $L M I$ (11) with $\overline{\mathrm{G}}=0$. Indeed, this case is straightforward by noticing that making $\overline{\mathrm{G}}=0$ imposes that $\mathrm{G}=0$, thus turning set $\mathcal{L}$ into the whole state-space.

\subsection{Optimization issues}

From (14), it becomes clear that minimization of $\gamma$ leads to minimization of the energy of $\operatorname{sat}\left(y_{f}(t)\right)$. Therefore, while solving the LMIs in Theorem 1 (or in Remark 6), we can accomplish better results for the allocator by minimizing $\gamma$. In case of Theorem 1, the maximization of the ellipsoid $\varepsilon(\mathrm{P}, 1)$ is also of interest. Therefore, a multi-objective optimization procedure applies. Consider a positive definite matrix $\mathrm{P}_{0}$ and the following matrix inequality

$$
\left[\begin{array}{cc}
\mathrm{P}_{0} & \mathrm{I} \\
\star & \overline{\mathrm{J}}+\overline{\mathrm{J}}^{\top}-\overline{\mathrm{P}}
\end{array}\right] \succeq 0 .
$$

Then, minimization of the trace of $\mathrm{P}_{0}$ indirectly leads to minimization of the trace of $\mathrm{P}$ and, therefore, to maximization of the ellipsoid $\varepsilon(\mathrm{P}, 1)$. Consider weighting parameters $\rho_{1}, \rho_{2}$. Then the following optimization procedure takes place in case of Theorem 1

$$
\begin{aligned}
& \min \rho_{1} \lambda+\rho_{2} \gamma \\
& \text { subject to (11), (12), (17), } \mathrm{P}_{0} \preceq \lambda \mathrm{I}
\end{aligned}
$$

In case global asymptotic stability is sought (Remark 6), the following optimization procedure applies

$$
\begin{aligned}
& \min \gamma \\
& \text { subject to (11) with } \overline{\mathrm{G}}=0
\end{aligned}
$$




\section{Simulation results}

\subsection{Example 1}

In this example we consider the exponentially stable plant from [15], where the saturation limits are given by $\bar{u}=\left[\begin{array}{lll}1 & 0.01 & 0.02\end{array}\right]^{\top}$. The plant is defined by the following data:

$$
\left[\begin{array}{c|c}
\mathrm{A}_{p} & \mathrm{~B}_{p} \\
\hline \mathrm{C}_{p} & \mathrm{D}_{p}
\end{array}\right]=\left[\begin{array}{cc|ccc}
-0.157 & -0.094 & 0.87 & 0.253 & 0.743 \\
-0.416 & -0.45 & 0.39 & 0.354 & 0.65 \\
\hline 0 & 1 & 0 & 0 & 0
\end{array}\right] .
$$

There is no loss of generality in considering this example since $\mathrm{D}_{p}=0$. To control the system and guarantee asymptotic tracking of constant references in the absence of saturation, [15] inserts an integrator and designs a stabilizing LQG controller which purposefully only uses the first two input channels. The resulting controller is given by

$$
\left[\begin{array}{c|c}
\mathrm{A}_{c} & \mathrm{~B}_{c} \\
\hline \mathrm{C}_{c} & \mathrm{D}_{c}
\end{array}\right]=\left[\begin{array}{cccc|c}
-1.57 & 0.5767 & 0.822 & -0.65 & 0 \\
-0.9 & -0.501 & -0.94 & 0.802 & 0 \\
0 & 1 & -1.61 & 1.614 & 0 \\
0 & 0 & 0 & 0 & -1 \\
\hline 1.81 & -1.2 & -0.46 & 0 & 0 \\
-0.62 & 1.47 & 0.89 & 0 & 0 \\
0 & 0 & 0 & 0 & 0
\end{array}\right] .
$$

For this example, $m_{a}=m_{c}$ and $\mathrm{M}=\mathrm{I}$. We select then $\mathrm{N}$ as the Kernel of $\mathrm{B}_{p}$, resulting in $\mathrm{N}=$ $\left[\begin{array}{lll}-0.4726 & -1.3143 & 1\end{array}\right]^{\top}$. The entries of matrix $\mathrm{W}$ are chosen as $w_{i}=\bar{u}_{(i)}^{-1}$. In this case, we utilize optimization procedure (19) which allows to establish global asymptotic stability results (Remark 6). We obtain, thus $\mathrm{K}_{f}=-2.4992$ and

$$
\left[\begin{array}{l}
\mathrm{E}_{c} \\
\hline \mathrm{E}_{f}
\end{array}\right]=\left[\begin{array}{ccc}
-0.8972 & -0.1642 & -0.7012 \\
-0.3176 & -0.3523 & -0.6356 \\
-0.5494 & 0.0361 & -0.0159 \\
-0.5607 & 0.2415 & 0.1140 \\
\hline-0.5958 & -0.0456 & -0.6322
\end{array}\right]
$$

The parameters of the allocator and the anti-windup of [15] can be found therein. We simulate the system response for an initial condition $x_{p}(0)=\left[\begin{array}{ll}0 & 1\end{array}\right]^{\top}$, with $x_{c}(0)=0$ and $x_{f}(0)=0$. Figure 2 shows the output response, the computed control signal and the plant input for both the proposed strategy and the one from [15]. The fundamental difference in the results relies in the fact that for the proposed strategy the plant input signals converge to the origin, thus avoiding waste of energy in the actuators.

\subsection{Example 2}

Consider the satellite formation flying control problem from [5], where the controlled output $y_{p}$ represents the relative position between two satellites in a vertical axis. Given two satellites, the objective is to cancel the lateral position error between them in the $z$-axis. The process can be represented by the following model

$$
\left[\begin{array}{c|c}
\mathrm{A}_{p} & \mathrm{~B}_{p} \\
\hline \mathrm{C}_{p} & \mathrm{D}_{p}
\end{array}\right]=\left[\begin{array}{cc|cc}
0 & 1 & 0 & 0 \\
0 & 0 & m_{1}^{-1} & -m_{2}^{-1} \\
\hline 1 & 0 & 0 & 0
\end{array}\right]
$$

where $m_{1}^{-1}$ and $m_{2}^{-1}$ are the masses of the two satellites. The plant input is given by $u_{p}=\left[\begin{array}{l}u_{p_{1}} \\ u_{p_{2}}\end{array}\right]=\left[\begin{array}{l}F_{1} \\ F_{2}\end{array}\right]$, where $F_{1}$ and $F_{2}$ are forces that act individually in each satellite. Each satellite possesses 4 thrusters that 

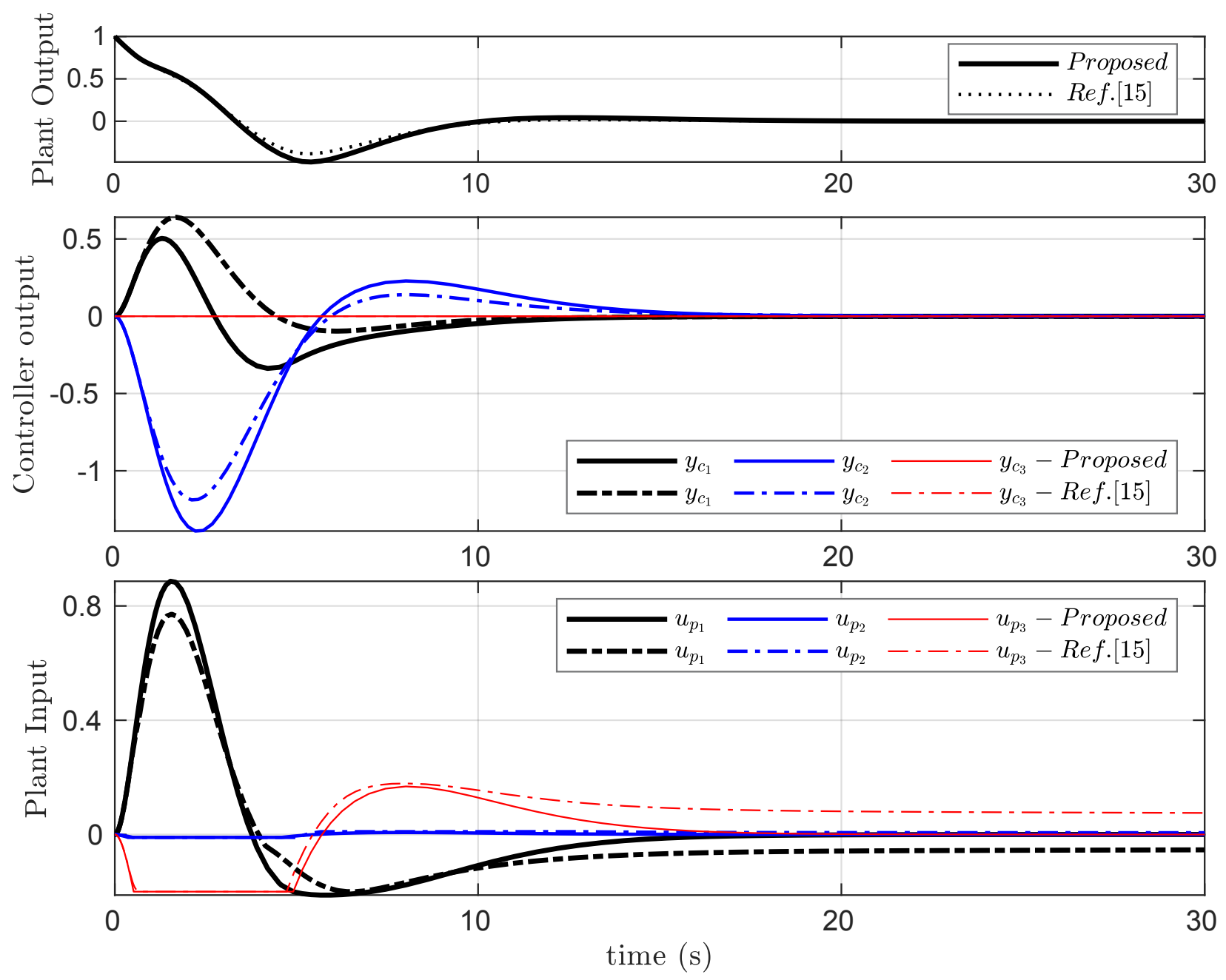

Figure 2: Example 1: Plant output, controller output and plant output signals for both the proposed strategy and $[15]$.

jointly produce the force applied in each of them. The influence matrix is given by $M=\left[\begin{array}{cc}M_{1} & 0 \\ 0 & M_{2}\end{array}\right]$, with $\mathrm{M}_{1}=\mathrm{M}_{2}=\left[\begin{array}{llll}1 & -1 & -1 & 1\end{array}\right]$. We assume that each thruster can produce a force between $0 \mathrm{mN}$ and $100 \mathrm{mN}$, therefore the saturation limits are not symmetric. In order to apply the developed conditions, the same symmetrizing technique of [5] takes place, consisting of substituting the asymmetric saturation by a symmetric one with limits $\bar{u}_{i}=50 m N, i=1, \ldots, 8$, followed by addition of the kernel symmetrizing vector $\xi=\bar{u}$. Note that the work in [5] explored the combination of the static allocator $\left(\mathcal{F}=\mathrm{M}^{\dagger}\right)$ with three different anti-windup strategies. Here we focalize on the co-design of the dynamic allocator $(\mathcal{F}$ given by $(6))$ and the controller anti-windup gain $\mathrm{E}_{c}$.

After choosing $m_{1}=m_{2}=1000 \mathrm{~kg}$, a stabilizing LQG controller is designed using identity matrices for all the weights. The resulting controller is given by

$$
\left[\begin{array}{c|c}
\mathrm{A}_{c} & \mathrm{~B}_{c} \\
\hline \mathrm{C}_{c} & \mathrm{D}_{c}
\end{array}\right]=\left[\begin{array}{cc|c}
-1.7321 & 1 & 1.7321 \\
-1.0014 & -0.0532 & 1 \\
\hline-0.7071 & -26.6009 & 0 \\
0.7071 & 26.6009 & 0
\end{array}\right]
$$




$$
\begin{gathered}
{\left[\begin{array}{c}
\mathrm{E}_{c} \\
\hline \mathrm{E}_{f}
\end{array}\right]=\left[\begin{array}{cccccccc}
0.0019 & -0.0000 & 0.0394 & -0.0193 & 0.0325 & -0.0411 & -0.0411 & 0.0411 \\
-0.0002 & -0.0047 & 0.0142 & -0.0043 & 0.0118 & -0.0160 & -0.0160 & 0.0160 \\
\hline 1.2781 & 0.0144 & 0.1663 & -0.0741 & 0.1006 & 0.1297 & 0.1297 & -0.1297 \\
-0.6243 & -0.0044 & -0.0738 & 0.3141 & 0.2881 & -0.0918 & -0.0918 & 0.0918 \\
0.7725 & 0.0088 & 0.0736 & 0.2114 & 0.3749 & 0.0720 & 0.0720 & -0.0720 \\
-0.9763 & -0.0119 & 0.0949 & -0.0674 & 0.0721 & 0.9519 & -0.3357 & 0.3357 \\
-0.9763 & -0.0119 & 0.0949 & -0.0674 & 0.0721 & -0.3357 & 0.9519 & 0.3357 \\
0.9763 & 0.0119 & -0.0949 & 0.0674 & -0.0721 & 0.3357 & 0.3357 & 0.9519
\end{array}\right],} \\
\mathrm{K}_{f}=\left[\begin{array}{cccccc}
-1.1684 & 0.6813 & -0.4766 & 0.0034 & 0.0034 & -0.0034 \\
0.7282 & -1.0438 & -0.3054 & 0.0249 & 0.0249 & -0.0249 \\
-0.4528 & -0.3418 & -0.8017 & 0.0284 & 0.0284 & -0.0284 \\
-0.0200 & 0.0792 & 0.0584 & -0.8628 & 0.1381 & -0.1381 \\
-0.0200 & 0.0792 & 0.0584 & 0.1381 & -0.8628 & -0.1381 \\
0.0200 & -0.0792 & -0.0584 & -0.1381 & -0.1381 & -0.8628
\end{array}\right]
\end{gathered}
$$

We then compute $\mathrm{M}^{\dagger}=0.25 \operatorname{diag}\left(\mathrm{M}_{1}^{\top}, \mathrm{M}_{2}^{\top}\right), \mathrm{N}=\operatorname{diag}\left(\mathrm{N}_{1}, \mathrm{~N}_{2}\right)$, with $\mathrm{N}_{1}=\mathrm{N}_{2}=\left[\begin{array}{ccc}1 & 1 & -1 \\ & \mathrm{I}_{3}\end{array}\right]$. We choose $\mathrm{W}=\operatorname{diag}(100,1, \ldots, 1)$ to illustrate the allocator ability, which means that we want to penalize the use of the first actuator. We use optimization procedure (18) with weights $\rho_{1}=1, \rho_{2}=0.15$, so that regional asymptotic stability can be guaranteed by means of Theorem 1 . To enlarge the region of stability in the direction of the first plant state, representing the distance between the satellites, we used a small modification in (18) by substitution of $\mathrm{P}_{0} \preceq \lambda \mathrm{I}$ by $\left[\begin{array}{ll}1 & 0_{1 \times(n-1)}\end{array}\right] \mathrm{P}_{0}\left[\begin{array}{ll}1 & 0_{1 \times(n-1)}\end{array}\right]^{\top} \preceq \lambda$. The obtained anti-windup and allocator matrices for this example are given in (20) and (21) (provided at the top of the page).

We simulate the system response for an initial condition of $x_{p}(0)=\left[\begin{array}{cc}-0.25 & 0\end{array}\right]^{\top}$, with $x_{c}(0)=0$ and $x_{f}(0)=0$. Figure 3 shows the obtained results. To better illustrate the ability of the dynamic allocator, two cases are plotted: dynamic allocator $\left(\mathcal{F}\right.$ defined in (6)) plus anti-windup gain $\left(\mathrm{E}_{c}\right)$ and static allocator $\left(\mathcal{F}=\mathrm{M}^{\dagger}\right)$ plus anti-windup gain $\left(\mathrm{E}_{c}\right)$. Both strategies are able to stabilize the systems, however it can be observed that the dynamic allocation successfully reduces the usage of the penalized actuator.

\section{Conclusion}

In this paper, we proposed the co-design of dynamic allocation functions along with anti-windup gains to deal with over actuated/input redundant systems with saturating actuators. The novelty of the article relied upon the extension of the ideas in [15] to a more general scenario, including the ability to deal with a much broader spectrum of cases and inserting optimization criteria that allow both to minimize energy consumption in the actuators and to maximize estimations on the region of attraction. The application of the developed conditions in the examples effectively showed the advantages of the proposed scheme. Future work envisages dealing with many other cases, as the consideration of other nonlinearities affecting the actuator and to deal with event-triggered control.

\section{References}

[1] Tor A. Johansen and Thor I. Fossen. Control allocation - a survey. Automatica, 49(5):1087 - 1103, 2013.

[2] Johannes Tjønnås and Tor A. Johansen. Adaptive control allocation. Automatica, 44(11):2754 - 2765, 2008. 

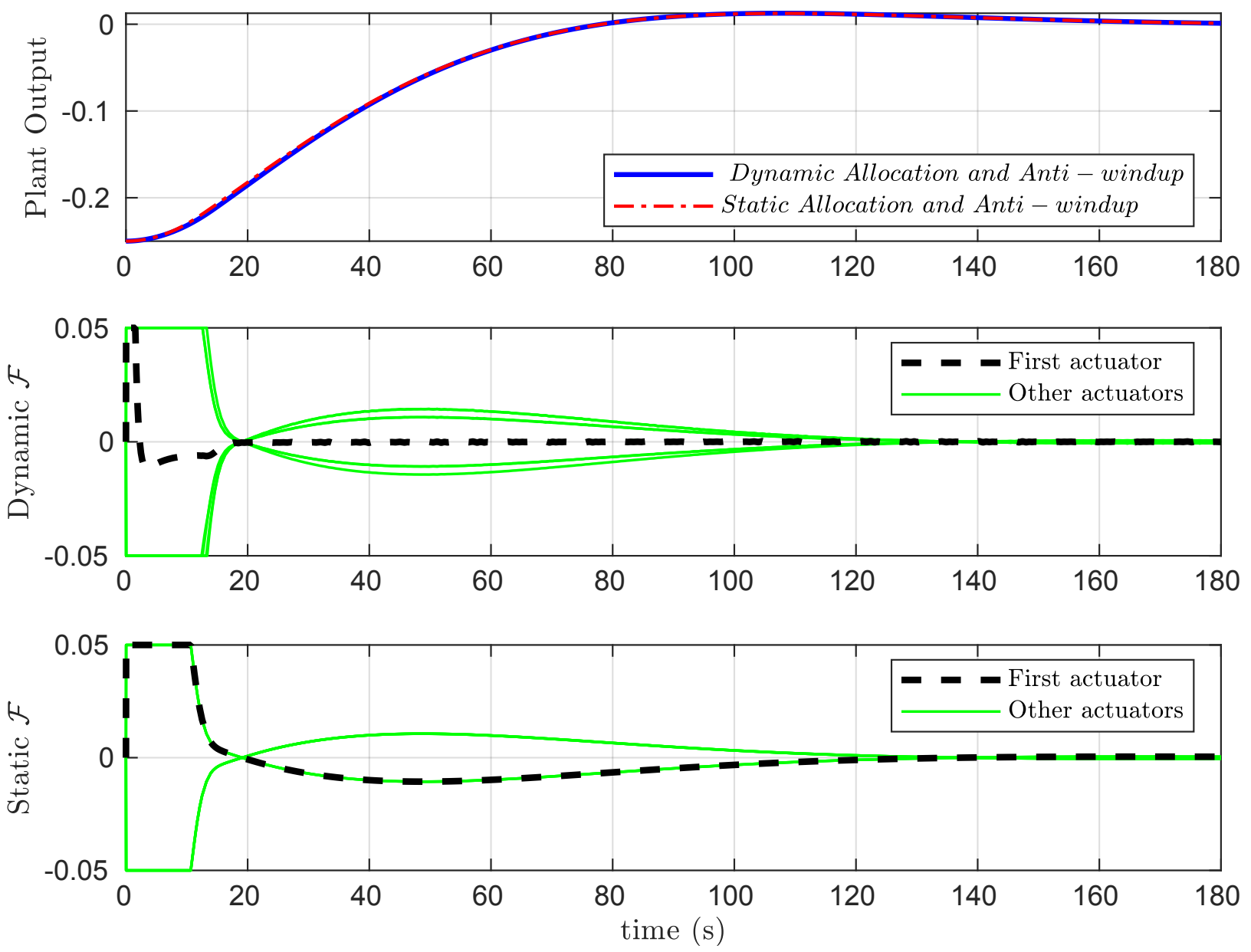

Figure 3: Example 2: Output and actuators .

[3] Jaehyun Jin. Modified pseudoinverse redistribution methods for redundant controls allocation. Journal of Guidance, Control, and Dynamics, 28(5):1076-1079, September 2005.

[4] M. W. Oppenheimer, D. B. Doman, and M. A. Bolender. Control allocation for over-actuated systems. In 2006 14th Mediterranean Conference on Control and Automation, pages 1-6, June 2006.

[5] Josep Boada, Christophe Prieur, Sophie Tarbouriech, Christelle Pittet, and Catherine Charbonnel. Formation flying control for satellites: Anti-windup based approach. In Giorgio Fasano and János D. Pintér, editors, Modeling and Optimization in Space Engineering, pages 61-83. Springer New York, New York, NY, 2013.

[6] Wayne Durham, Kenneth A. Bordignon, and Roger Beck. Aircraft Control Allocation. John Wiley \& Sons, Ltd, December 2016.

[7] Sergio Galeani, Andrea Serrani, Gianluca Varano, and Luca Zaccarian. On input allocation-based regulation for linear over-actuated systems. Automatica, 52:346 - 354, 2015.

[8] A. Serrani. Output regulation for over-actuated linear systems via inverse model allocation. In 2012 IEEE 51st IEEE Conference on Decision and Control (CDC), pages 4871-4876, Dec 2012. 
[9] J. A. M. Petersen and M. Bodson. Constrained quadratic programming techniques for control allocation. IEEE Transactions on Control Systems Technology, 14(1):91-98, Jan 2006.

[10] R. de Castro and J. Brembeck. Lyapunov-based control allocation for over-actuated nonlinear systems. In 2019 American Control Conference (ACC), pages 5033-5038, 2019.

[11] M. Benosman, F. Liao, K. Lum, and J. L. Wang. Nonlinear control allocation for non-minimum phase systems. IEEE Transactions on Control Systems Technology, 17(2):394-404, 2009.

[12] F. Liao, K. Lum, J. L. Wang, and M. Benosman. Constrained nonlinear finite-time control allocation. In 2007 American Control Conference, pages 3801-3806, 2007.

[13] T. A. Johansen. Optimizing nonlinear control allocation. In 43rd IEEE Conference on Decision and Control (CDC), pages 3435-3440 Vol.4, 2004.

[14] T. A. Lima, S. Tarbouriech, F. G. Nogueira, and B. C. Torrico. Energy-based design of dynamic allocation in the presence of saturating actuators. In Accepted for Proceedings of the the 24th International Symposium on Mathematical Theory of Networks and Systems, August 2021.

[15] Luca Zaccarian. Dynamic allocation for input redundant control systems. Automatica, 45(6):1431 - 1438, 2009.

[16] S. Tarbouriech, G. García, J. M. Gomes da Silva Jr., and I. Queinnec. Stability and Stabilization of Linear Systems with Saturating Actuators. Springer, London, 2011.

[17] Luca Zaccarian and Andrew R. Teel. Modern Anti-windup Synthesis: Control Augmentation for Actuator Saturation (Princeton Series in Applied Mathematics). Princeton University Press, 2011.

[18] E. D. Sontag. An algebraic approach to bounded controllability of linear systems. Int. J. Control, 39(1):181-188, 1984.

[19] Maurício C. de Oliveira and Robert E. Skelton. Stability tests for constrained linear systems. In S.O. Reza Moheimani, editor, Perspectives in robust control, pages 241-257, London, 2001. Springer London. 